OPEN ACCESS

\section{Sub-barrier transfer reactions studied with the magnetic spectrometer PRISMA}

To cite this article: E Fioretto et al 2014 J. Phys.: Conf. Ser. 533012006

View the article online for updates and enhancements.
You may also like

Exploring the evolution of the shell
structure by means of deep inelastic
reactions: recent results from LNL
Giacomo de Angelis
Spectroscopic studies with the PRISMA-
CLARA set-up
E Fioretto, D Bazzacco, S Beghini et al.
The Legnaro National Laboratories and
the SPES facility: nuclear structure and
reactions today and tomorrow
Giacomo de Angelis and Gianni Fiorentini

The Electrochemical Society

Advancing solid state \& electrochemical science \& technology

243rd Meeting with SOFC-XVIII

Boston, MA • May 28 - June 2, 2023

Accelerate scientific discovery!
Learn More \& Register

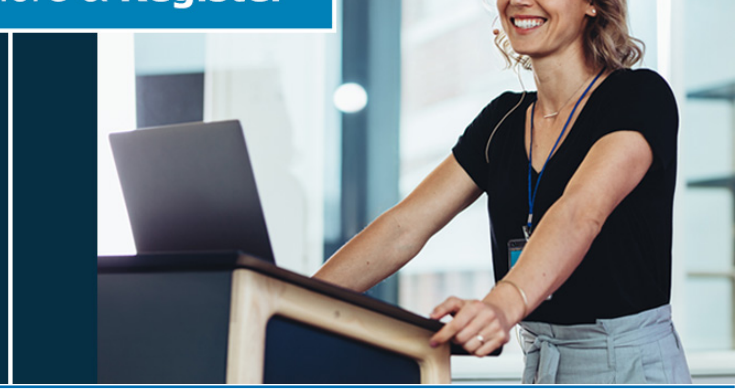

This content was downloaded from IP address 54.198 .187 .58 on 26/04/2023 at 14:13 


\title{
Sub-barrier transfer reactions studied with the magnetic spectrometer PRISMA
}

\author{
E Fioretto ${ }^{1}$, L Corradi ${ }^{1}$, S Szilner ${ }^{2}$, D Montanari ${ }^{3}$, C Michelagnoli $^{3}$, T Mijatović ${ }^{2}$,

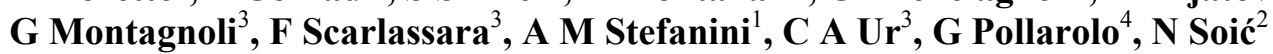 \\ ${ }^{1}$ INFN - Laboratori Nazionali di Legnaro, Viale dell'Università 2, Legnaro (PD), \\ I-35020, Italy \\ ${ }^{2}$ Ruđer Bošković Institute, Bijenička 54, Zagreb, HR-10001, Croatia \\ ${ }^{3}$ Dipartimento di Fisica dell'Università di Padova and INFN, Via Marzolo 8, Padova, \\ I-35131, Italy \\ ${ }^{4}$ Dipartimento di Fisica Teorica dell’Università di Torino and INFN, Via P. Giuria 1, \\ Torino, I-10125, Italy \\ E-mail: fioretto@lnl.infn.it
}

\begin{abstract}
Transfer reactions in the sub-barrier region have been studied at Laboratori Nazionali di Legnaro (LNL) by using the large solid angle magnetic spectrometer PRISMA. In particular, excitation functions of multinucleon transfer channels have been measured for the systems ${ }^{96} \mathrm{Zr}+{ }^{40} \mathrm{Ca}$ (closed shell nuclei), ${ }^{116} \mathrm{Sn}+{ }^{60} \mathrm{Ni}$ (super-fluid nuclei) from the Coulomb barrier to $25 \%$ below. Results of these measurements are presented in this contribution.

An ancillary detection system has been designed and built in order to perform kinematic coincidence measurements in conjunction with PRISMA. It will allow to study the effects of secondary processes, such as evaporation and fission, which strongly affect the final yield of the reaction products. The status of this development is also reported.
\end{abstract}

\section{Introduction}

Transfer reactions play an important role in both nuclear structure and reaction dynamics studies. Oneand two-nucleon transfer processes can be used to get useful information on the single particle structure and the pairing correlations in nuclei [1]. In the sub-barrier regime only few transfer channels are open and the Q-value distributions become narrower. These peculiar conditions should allow to extract more quantitative information on the mechanism of multiple transfer processes, in particular on the relative contribution of single particle and more complex degrees of freedom which include nucleon-nucleon correlations. However, available data for heavy ion transfer reactions in the subbarrier region are extremely scarce or almost not existing due to their significant experimental difficulties. The use of large acceptance magnetic spectrometers such as PRISMA $[2,3,4]$ and inverse kinematics reactions allows to perform this kind of experiments in an efficient way.

The study of the heavy neutron-rich nuclei is presently receiving a distinctive attention from the nuclear physics and nuclear astrophysics communities. One of the promising tool for the production of neutron-rich heavy nuclei is the use of multinucleon transfer reactions at energies close to the Coulomb barrier. Kinematic coincidence measurements are crucial to evaluate the production rate of both binary partners, and especially the relevance of secondary processes in population of exotic heavy neutron-rich nuclei. 
XX International School on Nuclear Physics, Neutron Physics and Applications (Varna2013) IOP Publishing Journal of Physics: Conference Series 533 (2014) $012006 \quad$ doi:10.1088/1742-6596/533/1/012006

\section{Sub-barrier transfer measurements}

Transfer processes have been investigated in a wide range of energies for the ${ }^{96} \mathrm{Zr}+{ }^{40} \mathrm{Ca}$ and ${ }^{116} \mathrm{Sn}+{ }^{60} \mathrm{Ni}$ inverse kinematics reactions. The ${ }^{96} \mathrm{Zr}$ and ${ }^{116} \mathrm{Sn}$ beams were delivered by the Tandem/PIAVE-ALPI accelerator complex of the Laboratori Nazionali di Legnaro at energies ranging from the Coulomb barrier down to $25 \%$ below (from $330 \mathrm{MeV}$ to $275 \mathrm{MeV}$ for ${ }^{96} \mathrm{Zr}$ - from $500 \mathrm{MeV}$ to $395 \mathrm{MeV}$ for ${ }^{116} \mathrm{Sn}$ ) with an average current of about $2 \mathrm{pnA}$. Light target-like ions ${ }^{40} \mathrm{Ca}$ and ${ }^{60} \mathrm{Ni}$ were detected at a very forward angle $\left(\theta_{l a b}=20^{\circ}\right)$ with the large acceptance magnetic spectrometer PRISMA. The use of inverse kinematics allowed to have at the same time target-like ions with enough kinetic energy and forward focused angular distributions resulting in good mass $A$ and atomic number $Z$ resolutions and high detection efficiency, respectively. Excitation functions have been measured for both systems and, making use of semi-classical conditions, transfer probabilities $P_{t r}$ have been extracted for the most intense transfer channels (with $P_{t r}$ defined as the ratio of transfer cross section to the elastic one). Experimental $P_{t r}$ are plotted as a function of the distance of closest approach $D$ in figure 1 for ${ }^{96} \mathrm{Zr}+{ }^{40} \mathrm{Ca}$ and in figure 2 for ${ }^{116} \mathrm{Sn}+{ }^{60} \mathrm{Ni}$. The experimental slopes agree well with those predicted by the binding energies of transferred nucleons. In both systems one finds two-neutron transfer probabilities higher than the simple product of probabilities for single neutron transfer, phenomenologically indicating degrees of freedom beyond independent particle transfer.

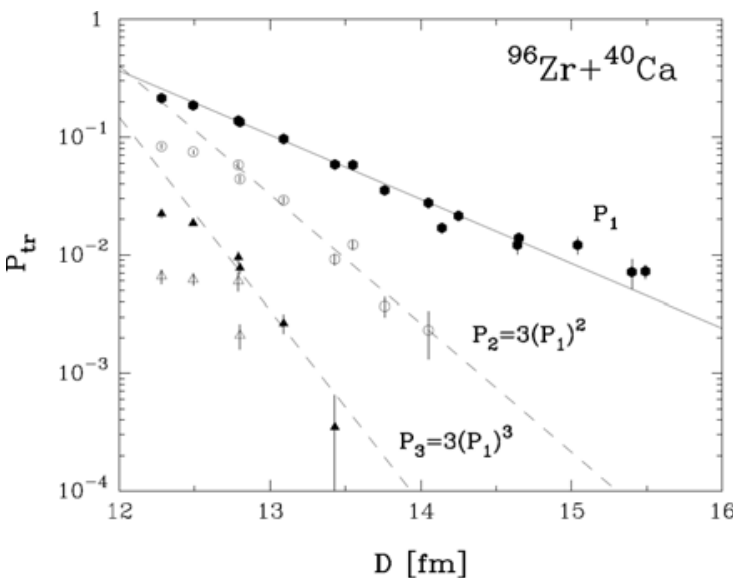

Figure 1. Transfer probabilities of $+1 n$ (full circles), $+2 n$ (empty circles), $+3 n$ (full triangles) and $+4 n$ (empty triangles) transfer channels, respectively. The full line is a fit of the data for the $+1 n$ pick-up channel according to the expression $P_{1 n}=\exp (13.95-1.25 D)$. The dashed lines correspond to the shown equations.

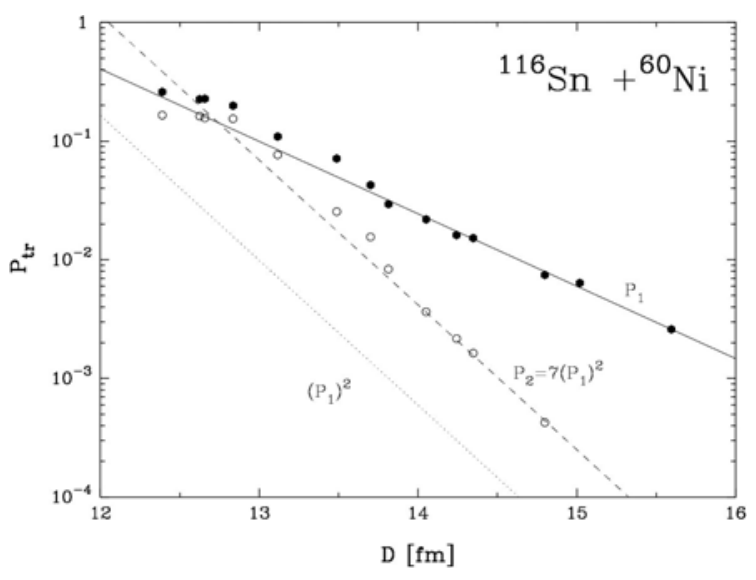

Figure 2. Transfer probabilities of $+1 n$ (full circles), $+2 n$ (empty circles) transfer channels, respectively. The full line is a fit of the data for the $+1 n$ pick-up channel according to the expression $P_{1 n}=\exp (13.86-1.22 D)$. For the sake of comparison $\left(P_{1}\right)^{2}$ (dotted line) is also indicated in the plot.

Closed or near-closed shell nuclei represent a good reference for a quantitative comparison with theoretical calculations. Thus, the experimental data for ${ }^{96} \mathrm{Zr}+{ }^{40} \mathrm{Ca}$ have been compared with microscopic calculations performed within a semi-classical theory [5], where for the two particle transfer the $0^{+}$states of both projectile and target have been included. Figure 3 shows the results of these calculations where the full line represents the inclusive transfer probability for $+1 n$ transfer channel, the dotted line the ground state to ground state transition for the $+2 n$ transfer and the dashed line the transition to the $0^{+}$excited state at $5.76 \mathrm{MeV}$ in ${ }^{42} \mathrm{Ca}$. From the comparison with the experimental data it turns out that the contribution to the cross section of this excited $0^{+}$state in ${ }^{42} \mathrm{Ca}$ is much larger than the contribution of the ground state. Still, the experimental transfer probability is underestimated by a factor $\sim 3$. We presently ascribe this enhancement to the fact that the two-nucleon 
transfer reaction does not populate only $0^{+}$states but it is much richer, so that more complicated twoparticle correlations have to be taken into account.

At variance with the ${ }^{96} \mathrm{Zr}+{ }^{40} \mathrm{Ca}$ system, in the ${ }^{116} \mathrm{Sn}+{ }^{60} \mathrm{Ni}$ system (super-fluid nuclei) [6] the ground to ground state Q-values for neutron transfers are close to zero matching their optimum Q-value. Figure 4 shows TKEL spectra for the elastic, the one $(+1 n)$ and the two $(+2 n)$ neutron pick-up channels at different bombarding energies. The vertical dashed lines indicate the position of the Q-value for the elastic which is taken as a reference for all the other isotopes. TKEL distributions for neutron transfers are peaked around $Q=0$, as it is expected. At the same time one sees a tail toward larger TKEL, more marked when more nucleons are transferred. These energy loss components tend to disappear far below the barrier. Microscopic calculations for this system are in progress and it will be interesting to see how calculations including only transfer of $J=0^{+}$pairs to $0_{g s}^{+}$compare with the experimental data, improving our understanding of the origin of the enhancement factors.

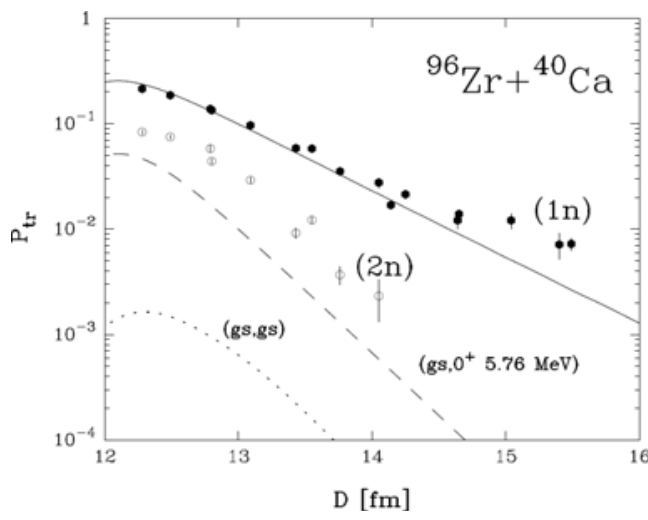

Figure 3. Theoretical transfer probabilities for one- and two-particle transfer (lines) in comparison with the experimental data (points).
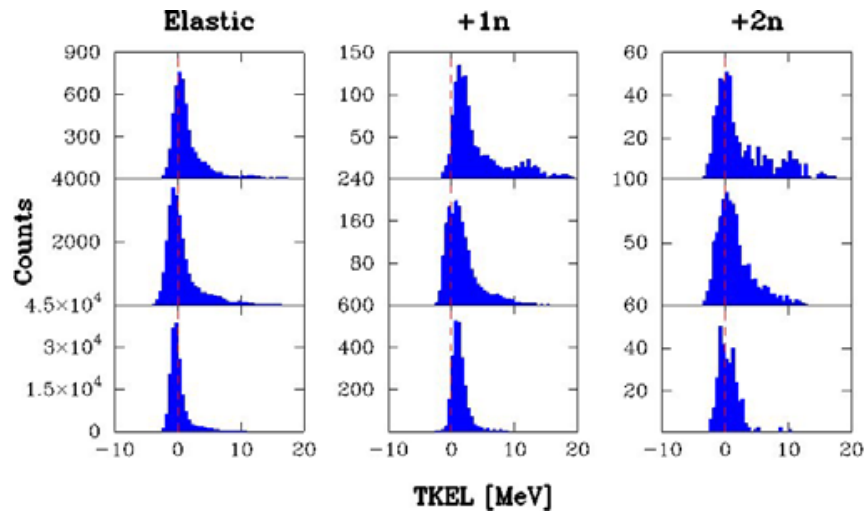

Figure 4. TKEL spectra for the elastic and $+1 n,+2 n$ transfer channels measured for the ${ }^{116} \mathrm{Sn}+{ }^{60} \mathrm{Ni}$ reaction at $\mathrm{E}_{\text {lab }}=500 \mathrm{MeV}$ (upper row), $\mathrm{E}_{\text {lab }}=460 \mathrm{MeV}$ (middle row) and $\mathrm{E}_{\text {lab }}=430 \mathrm{MeV}$ (lower row).

\section{The second arm of PRISMA}

Multinucleon transfer reactions attracted much interest in the last decade due to the fact that they can be used as a powerful tool to populate heavy neutron-rich nuclei in mass regions where production by other methods is very difficult. To predict the optimal conditions for the production of specific nuclei, one has to get quantitative information on the lowering of the final yields due to the onset of secondary processes (such as nucleon evaporation and fission) of which the heavy partner is generally much more affected. An important step forward in this kind of experiments is the detection of both partners participating in the reaction. In order to perform kinematic coincidence measurements in conjunction with the magnetic spectrometer PRISMA a new detection system has been built at LNL.

It is mainly composed of a position sensitive Parallel Plate Avalanche Counter (PPAC) followed by an axial field ionization chamber also known as Bragg chamber (BC). The PPAC consists of two anode planes (10 $\mu \mathrm{m}$ diameter and $1 \mathrm{~mm}$ spacing) and a central cathode made of an aluminized mylar foil ( $1.5 \mu \mathrm{m}$ thick and $20 \mu \mathrm{g} / \mathrm{cm}^{2} \mathrm{Al}$ on both sides). The distance cathode-anodes is $2 \mathrm{~mm}$. The detector has an active area of $10 \times 10 \mathrm{~cm}^{2}$ and provides a timing signal for Time of Flight measurements and $X$ and $Y$ position information (delay-line readout).

The $\mathrm{BC}$ has a $32 \mathrm{~cm}$ active depth with a Frish grid (FG) to anode distance of $2 \mathrm{~cm}$. The voltages for the 114 guard rings between the cathode and the FG are reduced by means of voltage dividers made of $10 \mathrm{M} \Omega$ resistors. As well known, axial field ionization chambers allow to measure the charge distribution produced along the ionization track (Bragg curve) and to perform a Bragg Curve Spectroscopy (BCS) first proposed and tested by Gruhn et al [7]. 
XX International School on Nuclear Physics, Neutron Physics and Applications (Varna2013) IOP Publishing Journal of Physics: Conference Series 533 (2014) $012006 \quad$ doi:10.1088/1742-6596/533/1/012006

Preliminary bench-tests of the detectors have been performed in laboratory with $\alpha$ particles from a mixed ${ }^{239} \mathrm{Pu}^{241} \mathrm{Am}^{244} \mathrm{Cm}$ source. In these tests the classical method of analog readout of the Bragg chamber was used: a charge sensitive preamplifier followed by two spectroscopy amplifiers in parallel, each with different shaping times $\tau$ ( 4 and $0.5 \mu$ s, respectively). Typical two-dimensional scatter-plot Bragg peak (BP) vs $E$ is shown in figure 5. An energy resolution of about $2 \%$ and position resolutions of $1 \mathrm{~mm}$ in both $X$ (figure 6) and $Y$ directions have been obtained, respectively. In-beam tests of this second arm will be carried out at LNL in the forthcoming months with different heavy ion beams. The use of digital techniques via a Flash ADC and the development of specific algorithms should improve the identification of the heavy binary partners providing the possibility of extracting additional parameters (such as the range $R$ and the energy loss $\Delta E$ ) from the Bragg curve.

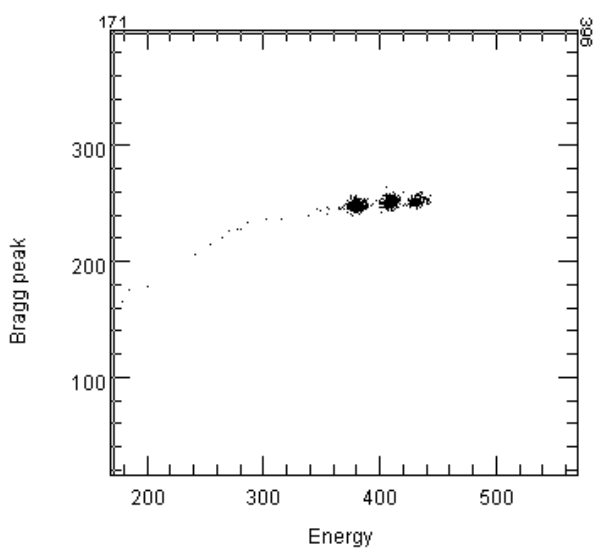

Figure 5. Two-dimensional scatter-plot BP (arb. units) vs $E$ (arb. units) obtained with a mixed $\alpha$ source.

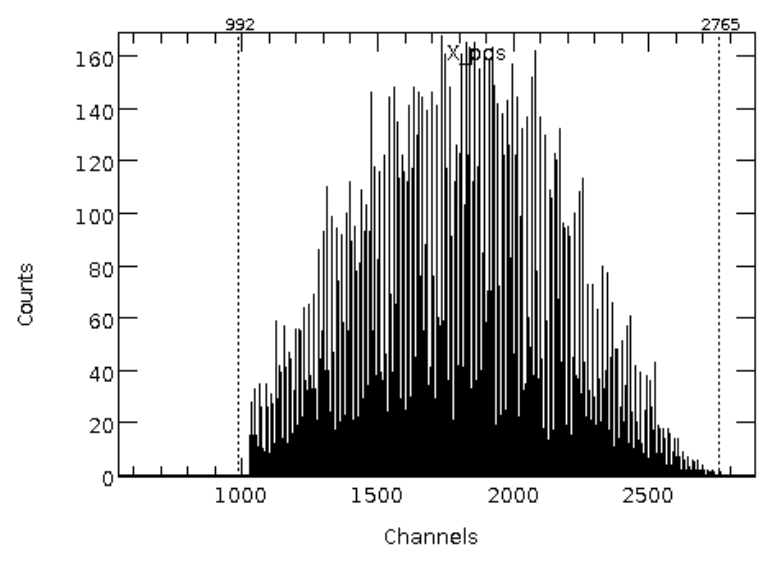

Figure 6. $X$ position spectrum measured with the PPAC mounted in front of the axial ionization chamber.

\section{Summary}

We measured excitation functions for the ${ }^{96} \mathrm{Zr}+{ }^{40} \mathrm{Ca}$ and ${ }^{116} \mathrm{Sn}+{ }^{60} \mathrm{Ni}$ inverse kinematics reactions. Light target-like ions have been detected with the magnetic spectrometer PRISMA with a good mass and atomic number resolution in the whole energy range. Enhancement of the two-neutron transfer has been evidenced for both systems. Microscopic calculations for the ${ }^{116} \mathrm{Sn}+{ }^{60} \mathrm{Ni}$ system are in progress. The comparison between data and theory for both cases, namely near closed shell and super-fluid nuclei, will significantly improve our understanding of nucleon-nucleon correlations in the transfer process. A second arm of the PRISMA spectrometer has been built and tested in laboratory with $\alpha$ particles obtaining good energy and position resolutions. In-beam tests will allow to evaluate its $Z$ resolving power and to improve the identification of the heavy binary partners.

\section{Acknowledgements}

The research leading to these results has received funding from the European Union Seventh Framework Programme FP7/2007- 2013 under Grant Agreement No. 262010 - ENSAR.

\section{References}

[1] Corradi L et al 2009 J. of Phys. G 36113101

[2] Stefanini A M et al 2002 Nucl. Phys. A $701217 \mathrm{c}$

[3] Montagnoli G et al 2005 Nucl. Instr. and Meth. A 547455

[4] Beghini S et al 2005 Nucl. Instr. and Meth. A 551364

[5] Corradi L et al 2011 Phys. Rev. C 84034603

[6] Montanari D et al 2013 INPC2013 (Firenze, Italy, 2-7 June 2013), ed. Signorini C et al in press

[7] Gruhn C R et al 1982 Nucl. Instr. and Meth. 19633 